\title{
Revisando el constructo articulación trabajo-familia: entre el conflicto y el enriquecimiento
}

\section{Work-family interface, revision of findings: between conflict and enrichment}

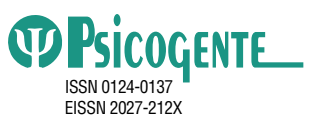

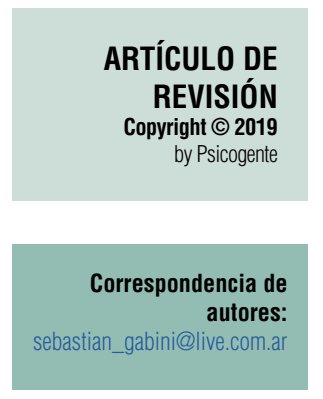

Recibido: $13-03-18$ Aceptado: $16-02-19$ Publicado: 01-07-19

\author{
Sebastián Gabini iD \\ Universidad Nacional de Rosario, Rosario, Argentina \\ CONICET, Rosario, Argentina \\ Universidad Abierta Interamericana, Rosario, Argentina
}

Resumen

Objetivo: El presente artículo de revisión tuvo como objetivo proporcionar una puesta al día acerca de la teoría e investigación sobre articulación trabajo-familia.

Método: Se realizó una búsqueda bibliográfica de artículos a texto completo correspondiente a los últimos cinco años (2011-2016) en bases de datos definidas como Grupo 1 (calidad AAA) por el Conicet, utilizando los descriptores: articulación trabajo-familia, conflicto trabajo-familia, enriquecimiento trabajo-familia. Luego de delimitar los resultados en función de determinados criterios de selección, se trabajó con un total de 54 artículos.

Resultados: La revisión realizada permitió sistematizar los principales mecanismos de vinculación de la interfase trabajo-familia, sus dimensiones constitutivas, las variables antecedentes y consecuentes, así como los instrumentos de medición desarrollados para evaluarla. De la misma surge que la literatura sobre el constructo ha estado dominada por la perspectiva del conflicto. A su vez, la inexistencia de un marco teórico integrador para el aspecto positivo de la articulación trabajo-familia ha dificultado las investigaciones en el área.

Conclusiones: La naturaleza del fenómeno debe ser investigada a partir de la evaluación conjunta de ambos aspectos de la articulación trabajo-familia. Futuras investigaciones podrían avanzar hacia una tipología que incorpore las diversas combinaciones de CTF y ETF, ampliar los alcances del modelo para verificar su validez transcultural y validar instrumentos con adecuadas propiedades psicométricas para tales fines.

Palabras clave: articulación trabajo-familia, conflicto trabajo-familia, enriquecimiento trabajo-familia, mecanismos de vinculación.

\section{Abstract}

Objective: This revision paper aims to provide an update on theory and research focus on work-family interface.

Method: A bibliographic research of academic full text articles over the past five years (2011-2016), in databases defined as Group 1 (AAA quality) by Conicet, using work-family interface, work-family conflict, work-family enrichment as descriptors, was made. After delimitating results according to certain selection criteria, we worked with a total of 54 papers.

Results: The revision allowed to identify principal mechanisms linking work- family interface, its constitutive dimensions, antecedent and consequent variables, as well as different and usefull assessment tools developed for evaluation. This revision shows that work-family literature has been dominated by a conflict perspective. In addition, the absence of a comprehensive theoretical framework to examine the positive effects of work and family interface, has prevented related researches in this area.

Conlusions: The nature of the construct should be investigated based on the specific combinations between work-family conflict and work-family enrichment. Future research could move towards a typology that incorporates different combinations of WFC and WFE, extend the scope of the model to verify its transcultural validity and validate instruments with adequate psychometric properties for such purposes.

Key words: work-family interface, work-family conflict, work-family enrichment, linking mechanisms.

Cómo citar este artículo (APA):

Gabini,S.(2019).Revisandoelconstructoarticulacióntrabajo-familia:entreelconflictoyelenriquecimiento.Psicogente22(42),1-26.https://doi.org/10.17081/psico.22.42.3470 


\section{INTRODUCCIÓN}

En las últimas décadas, el crecimiento del número de mujeres trabajadoras, la emergencia de parejas donde ambos cónyuges trabajan en el mercado laboral externo y el incremento de familias monoparentales, han provocado alteraciones en los enfoques tradicionales de la organización de la vida laboral y familiar (Gabini, 2017). Ante esta nueva realidad, muchos empleados se han visto sumergidos en el desafío de balancear sus roles familiares y laborales (Allen, Johnson, Kiburz, \& Shockley, 2013). Consecuentemente, la posibilidad de articular estas esferas vitales se ha transformado en un objeto de estudio primordial para los investigadores de la psicología organizacional, lo que ha generado un cuerpo sustancial de literatura específica sobre la temática.

En un primer momento, la investigación referida a esta problemática ha estado dominada por una perspectiva ligada al conflicto (Greenhaus \& Powell, 2006). De esta manera, el conflicto trabajo-familia (CTF) ha sido delimitado como la interferencia que ocurre entre los roles familiares y laborales, resultante de las presiones y demandas asociadas a cada dominio y que, además, puede llegar a tener efectos negativos sobre ambas esferas (Buonocore \& Russo, 2013).

Sin embargo, otros estudiosos de la temática (Lingard, Francis, \& Turner, 2010) se han mostrado escépticos de la perspectiva del conflicto y han sugerido que las ventajas de participar simultáneamente en diversos roles equilibrarían las desventajas generadas entre estos dominios. A pesar de ello, las primeras investigaciones realizadas en este sentido se han visto obstaculizadas por la carencia de claridad conceptual y por la falta de un marco teórico general e integrador (McNall, Masuda, Shanock, \& Nicklin, 2011). Este desacuerdo en relación a la vertiente positiva de la interacción trabajo-familia, se ha traducido en la utilización de diversas designaciones para señalar al constructo tales como derrame positivo, mejoramiento o facilitación (Omar, Urteaga, \& Salessi, 2015).

Con base en las dificultades provenientes de la falta de una denominación unívoca del fenómeno, Greenhaus y Powell (2006) propusieron el término enriquecimiento trabajo-familia (ETF) en referencia al grado en el que las experiencias vividas en un rol contribuyen a mejorar la calidad de vida en otros roles. En este sentido, recientemente se ha verificado (Daniel \& Sonnentag, 2016; Jijena-Michel \& Jijena-Michel, 2015; Nicklin \& McNall, 2013; Rastogi, Rangnekar, \& Rastogi, 2016) que las políticas organizacionales amigables con 
la familia cumplen un papel preponderante a la hora de enriquecer los roles entre ambos dominios.

Teniendo en cuenta la importancia del constructo, la que se ha visto reflejada en el aumento significativo de estudios relacionados a la temática en las últimas décadas (Allen, French, Dumani, \& Shockley, 2015), el objetivo del presente trabajo fue proporcionar una puesta al día sobre la teoría e investigación sobre articulación trabajo-familia a través de una revisión sistemática. Las mismas, son estudios de tipo secundario que tienen como fin resumir, compilar, criticar y sintetizar la investigación existente sobre un área temática o fenómeno de interés mediante un proceso de búsqueda, catalogación, ordenamiento y análisis (Perestelo-Pérez, 2013).

\section{MÉTODO}

\subsection{Diseño}

La presente investigación se enmarca en los lineamientos de la revisión bibliográfica sistemática. Se trata de un tipo de estudio exploratorio-descriptivo retrospectivo (Ato, López, \& Benavente, 2013).

\subsection{Procedimiento}

Se realizó una búsqueda bibliográfica con base en dos fases diferenciadas. La primera se centró en la revisión de artículos a texto completo correspondiente a los últimos cinco años (2011-2016) utilizando los descriptores: articulación trabajo-familia (work-family interface), conflicto trabajo-familia (work-family conflict), enriquecimiento trabajo-familia (work-family enrichment). Para tal fin se consultaron bases de datos definidas como Grupo 1 (calidad AAA) por el Conicet: Scopus, ISI, Social Sciences Citation Index, Scimago, Thomson Reuters, Web of Sciences y Scielo. En esta instancia se recuperaron 132 trabajos a texto completo. En la segunda fase, se seleccionaron aquellos resúmenes que cumplieran con los siguientes criterios: a) que el estudio se haya realizado en el marco de la psicología organizacional, b) que el uso de los descriptores fuera el utilizado por esta corriente psicológica, y c) que no formara parte de notas editoriales o comentarios generales de actividades sobre el tema.

\subsection{Análisis de la información}

En definitiva, se trabajó con un total de 54 artículos a texto completo sobre los cuales se realizaron análisis bibliométricos y de contenido. El análisis bibliométrico permitió obtener información cuantitativa sobre las investiga- 
ciones en un área de conocimiento. Se analizó la información obtenida de la búsqueda organizándola según los siguientes apartados: nombre de la publicación, año, tipo de artículo y abordaje de la articulación trabajo-familia. Por su parte, el análisis de contenido apuntó a describir el contenido manifiesto de las comunicaciones científicas recuperadas.

\subsection{Conflicto de interes}

No existe conflicto de interes en la ejecución y publicación de este estudio.

\section{RESULTADOS}

Los criterios de selección permitieron delimitar 54 artículos recuperados a partir de las búsquedas biblográficas realizadas. Los mismos fueron organizados según los parámetros y procedimiento expuestos anteriormente. En cuanto al año de publicación, la concentración de trabajos es mayor en el periodo 2015-2016 que en los periodos comprendidos entre los años 2011-2012 y 2013-2014. En lo que hace al número de estudios que contemplan el CTF o el ETF, de manera individual o conjunta, la cantidad de publicaciones es equitativa. Sin embargo, el análisis del abordaje de la temática muestra un mayor número de artículos que sólo evalúan el CTF entre 2011 y 2013, mientras que entre los años 2014 y 2016 existe una mayor preponderancia de estudios con foco en el ETF. Por su parte, el abordaje conjunto del CTF y el ETF ha sido más estable a través de los años (Figura 1).

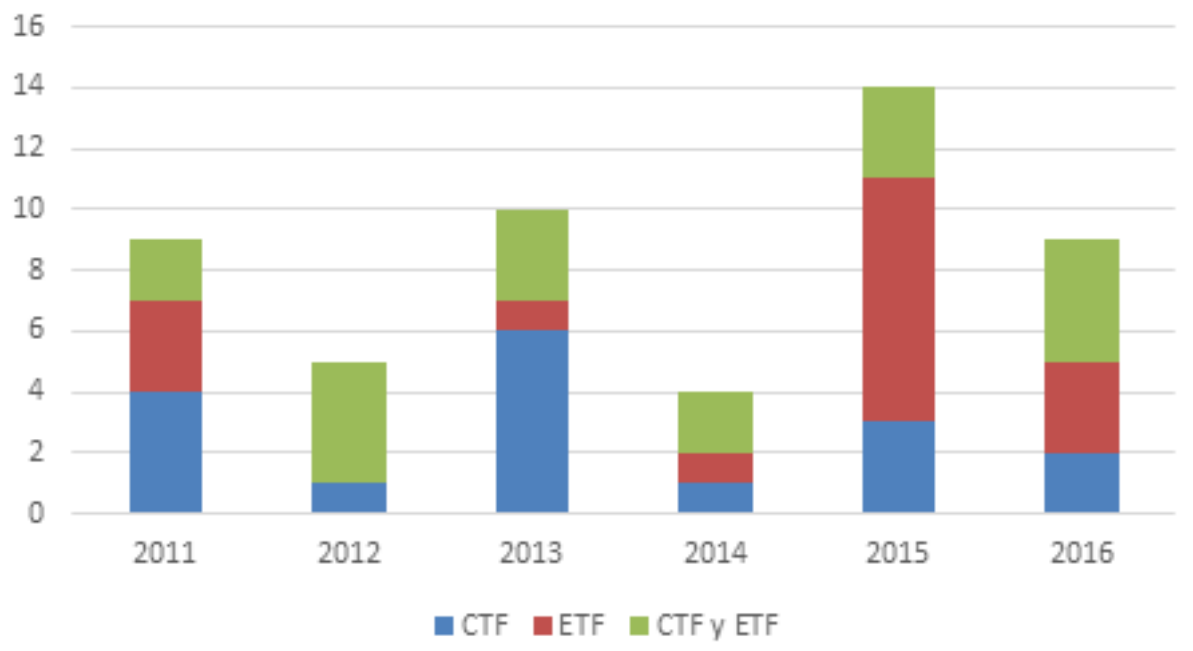

Figura 1. Distribución de las publicaciones por año y en función del abordaje (elaboración propia). 
En la Figura 2 se exhiben los resultados en relación al tipo de artículo recuperado. Los mismos muestran que el $87 \%$ de los trabajos revisados son de índole empírica, el $7 \%$ se trata de investigaciones de carácter instrumental, es decir que apuntan al desarrollo o validación de escalas de medición, y el restante $6 \%$ refiere a revisiones o estudios meta-analíticos.

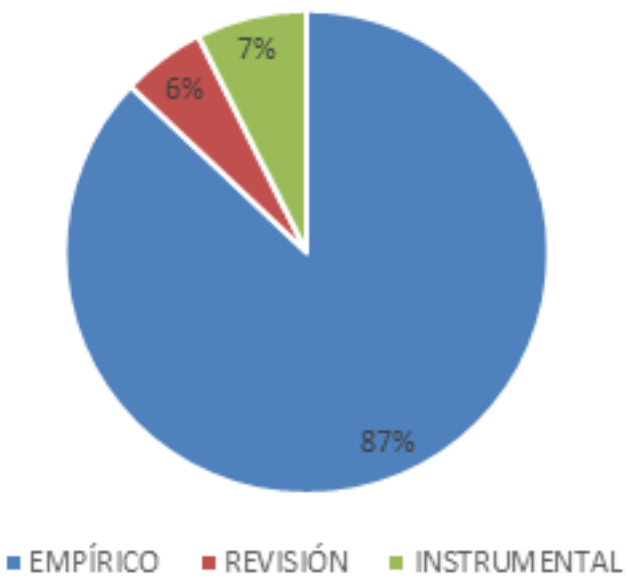

Figura 2. Tipos de artículos sobre articulación trabajo-familia (elaboración propia).

Un análisis pormenorizado de la información recuperada permitió identificar los principales mecanismos de vinculación de la interfase trabajo-familia, sus dimensiones constitutivas, las variables antecedentes y consecuentes del constructo, así como los instrumentos de medición desarrollados para evaluarla. A continuación se realiza una breve descripción de cada uno de estos aspectos.

\subsection{Mecanismos de articulación trabajo-familia}

Algunas décadas atrás, en las sociedades industrializadas, la vida familiar y laboral podían ser vistas como dos esferas independientes. No obstante, hoy en día los investigadores han aceptado el hecho que estos dos dominios se influyen mutuamente, lo que posibilita el intercambio de energía desde un rol al otro (Carlson, Hunter, Ferguson, \& Whitten, 2014). A partir de ello, han surgido al menos seis mecanismos explicativos tradicionales para dar cuenta de los intercambios que se producen entre ambas esferas (Edwards \& Rothbard, 2000) mientras que los modelos contemporáneos han comenzado a examinar la relación trabajo-familia de manera bilateral.

El mecanismo del derrame es uno de los primeros identificados en la bibliografía específica (Staines, 1980). El mismo hace referencia a los efectos que 
el trabajo o la familia tienen sobre el otro y que genera similitudes entre ellos. Estas similitudes usualmente son descritas en términos de afecto, valores, habilidades y comportamientos de apertura. En segunda instancia, el mecanismo de compensación (Champoux, 1978) hace hincapié en los esfuerzos para nivelar la insatisfacción en uno de los dominios a través de la búsqueda de la satisfacción en el otro. En tercer lugar, el mecanismo de segmentación (Piotrkowski, 1979) incorpora la posibilidad de separar la vida familiar de la vida laboral para que no se vean afectadas entre ellas. Cuarto, la reducción de recursos (Burke \& Greenglass, 1987) indica una relación negativa entre el trabajo y la familia, habida cuenta que el uso de recursos finitos (tiempo, energía o atención) en un dominio reduce su disponibilidad para el uso en otro dominio de la vida. Este mecanismo se diferencia del de la compensación, en el sentido que conlleva el cambio de recursos intencionales de un dominio al otro, debido a que esta transferencia no se realiza activamente (Burke \& Greenglass, 1987). En quinto lugar, el mecanismo de congruencia (Morf, 1989), refiere a la similitud entre el trabajo y la familia debido a una tercera variable que actúa como causa común. Estas causas comunes incluyen rasgos de personalidad, estilos de comportamiento, fuerzas sociales y culturales. Por último, el modelo de conflicto (Greenhaus \& Beutell, 1985) asume que participar en múltiples roles puede generar distracciones y agotar recursos, lo que tendría como resultado tensiones y sobrecargas para el trabajador.

Estas conceptualizaciones, tal cual fueron descritas, presentan importantes ambigüedades que han impedido la extrapolación de estos mecanismos de vinculación a estamentos teóricos precisos, lo que dificulta la posibilidad de definir si los hallazgos dan cuenta de algún proceso de vinculación en particular (Edwards \& Rothbard, 2000). En función de ello, las perspectivas posteriores (Ashforth, Kreiner, \& Fugate, 2000; Clark, 2000; Edwards \& Rothbard, 2000; Frone, 2003) han comenzado a desarrollar una mirada integrativa sobre la articulación trabajo-familia.

Una de las primeras perspectivas integrativas que abordó la problemática fue representada por el modelo de los límites entre roles y el proceso dinámico de la transición de roles (Ashforth et al., 2000; Clark, 2000). Al respecto, se propuso que cualquier par de roles puede ser dispuesto a lo largo de un continuum comprendido desde la alta segmentación hasta la alta integración. En efecto, dos roles se encuentran segmentados cuando las identidades de uno de ellos exhiben alto contraste y sus límites se muestran inflexibles e 
impermeables. En contraposición, dos roles están integrados cuando las identidades exhiben bajo contraste y sus límites son flexibles y permeables.

Por su parte, Edwards y Rothbard (2000) además del conflicto, optaron por incorporar la facilitación al modelo de articulación trabajo-familia. Desde este punto de vista, la articulación se erigía como un constructo multidimensional que necesitaba ser definido en relación a la dirección de la influencia entre los roles familiares y laborales, y al tipo de efecto generado entre ellos. Finalmente, siguiendo la perspectiva positiva de la interfase trabajo-familia, Greenhaus y Powell (2006) desarrollaron un marco teórico comprensivo al que denominaron ETF. Para este modelo, las experiencias en un rol ofrecen una serie de recursos que pueden ser adquiridos y trasladados a otro rol. Por ejemplo, si un empleado desarrolla habilidades multitarea a partir de su papel como padre, las mismas podrían aumentar su rendimiento laboral.

\subsection{Dimensiones de la articulación trabajo-familia}

El análisis de las investigaciones revisadas permite entrever que la perspectiva negativa (Burke \& Greenglass, 1987; Greenhaus \& Beutell, 1985) y positiva (Edwards \& Rothbard, 2000; Greenhaus \& Powell, 2006) de la articulación trabajo-familia han tenido un desarrollo independiente en la literatura específica. Sin embargo, la realidad indica que los individuos experimentan ambos fenómenos de manera simultánea (Paddhi \& Pattnaik, 2013). Por lo tanto, no debieran ser estudiados como compartimentos estancos sin interacción o influencia entre ellos, ya que ninguno de estos mecanismos aislados provee una base conceptual sólida para comprender la dinámica de la articulación entre ambas esferas (Frone, 2003). Como consecuencia, hace más de una década, algunos estudiosos de la temática (Bakker \& Geurts, 2004; Geurts et al., 2005; Michel \& Clark, 2009; Voydanoff, 2004) han comenzado a señalar que la naturaleza de la interacción trabajo-familia debe investigarse a partir de las combinaciones específicas entre estas dos perspectivas.

La necesidad de definir los modos en que el aspecto positivo y el negativo se relacionan, es decir si se trata de constructos isomorfos (aspectos opuestos de un continuum con los mismos antecedentes y consecuencias) u ortogonales (dimensiones distintas e independientes con antecedentes y consecuencias particulares). Al respecto, los resultados provenientes de un importante cúmulo de investigaciones empíricas (Bakker \& Geurts, 2004; Grzywacz \& Marks, 2000; Michel \& Clark, 2009; Voydanoff, 2004) han confirmado la pertenencia a este segundo grupo. A partir de estos avances, otras investigaciones recientes (Padhi \& Pattnaik, 2013; Rantanen, Kinnunen, Mauno \& 
Tement, 2013; Romeo, Berger, Yepes-Baldó, \& Ramos, 2014) han comenzado a llenar un área de vacancia en la temática, al centarse en el estudio de los aspectos positivos y negativos de manera conjunta, como parte del abordaje holístico de la interacción trabajo-familia. Esta perspectiva usualmente ha recaído (Powell \& Greenhaus, 2010; Rantanen et al., 2013) sobre los conceptos conflicto trabajo-familia (CTF) y enriquecimiento trabajo-familia (ETF) como términos genéricos. Por lo tanto, a continuación, se desarrollan ambos factores como dimensiones constitutivas del constructo articulación trabajo-familia:

\subsection{Conflicto trabajo-familia (CTF)}

La perspectiva negativa de la articulación trabajo-familia, el CTF, es un modo de conflicto entre roles en el cual las demandas generadas en un dominio son incompatibles con las del otro dominio. Por lo tanto, cumplimentar las exigencias de uno de ellos dificulta su concreción en el otro (Greenhaus \& Beutell, 1985). Siguiendo esta definición, el CTF puede ser delimitado en términos de su direccionalidad (Frone, 2003), es decir, en función de la orientación en la que se genera (Padhi \& Pattnaik, 2013) o sea, si parte de la familia hacia el trabajo (conflicto familia-trabajo, CFT) o desde el trabajo hacia la familia (conflicto trabajo-familia, CTF).

Paralelamente, se distinguieron tres formas de CTF: a) basado en el tiempo, ocurre cuando el tiempo destinado a cubrir las demandas de un dominio consume el tiempo necesario para cumplir las de otro dominio; b) basado en las tensiones, aparece cuando las tensiones tales como insatisfacción, ansiedad o fatiga que se generan en una faceta dificultan cumplir con las demandas de la otra, y c) basado en los comportamientos, se observa cuando los comportamientos desarrollados en un dominio son incompatibles con las demandas de los roles de otro dominio y, además, la persona no puede ajustarlos cuando se traslada entre las diferentes esferas de la vida (Carlson, Kacmar \& Williams, 2000).

\subsection{Enriquecimiento trabajo-familia (ETF)}

Hace poco más de una década, los investigadores de la temática (Greenhaus \& Powell, 2006; Carlson, Kacmar, Wayne, \& Grzywacz, 2006) se han preocupado por desarrollar un enfoque que conceda crédito a los efectos positivos de combinar ambos roles. De esta manera, desde su aparición en la bibliografía específica, el ETF ha comenzado a posicionarse en el lugar que ostenta hoy en día, es decir, como una de las dimensiones significativas de la articulación 
trabajo-familia (Crain \& Hammer, 2013). El constructo brinda, al igual que el CTF, la posibilidad de centrarse tanto en los efectos positivos del trabajo hacia la familia (enriquecimiento trabajo-familia, ETF), como de la familia hacia el trabajo (enriquecimiento familia-trabajo, EFT). Entre los recursos que pueden ser trasladados de un rol al otro (Crain \& Hammer, 2013) se incluyen habilidades (interpersonales, de afrontamiento), recursos físicos y psicológicos (autoeficacia, optimismo), capital social (información, trabajos en red), flexibilidad y recursos materiales (dinero, regalos).

A su vez, el ETF (Greenhaus \& Powell, 2006; Carlson et al., 2006) especifica dos mecanismos o caminos a partir de los cuales se realiza la transferencia de recursos: la vía instrumental o emocional. La ruta instrumental, también llamada directa, se identifica en situaciones en las cuales el trabajador cree que su vida familiar le ha enseñado nuevas formas de interactuar con los colegas o ha mejorado su capacidad para realizar múltiples tareas en el trabajo (Ruderman, Ohlott, Panzer, \& King, 2002). La ruta afectiva, por su parte, indica que la mayor atención en un dominio se relaciona indirectamente con mayor implicación en otro, a través del afecto positivo. Por ejemplo, una persona con buen humor y actitud después del trabajo probablemente responderá de forma positiva, paciente y agradable a los miembros de la familia, lo que en última instancia puede mejorar su desempeño o afecto como padre/madre y/o esposo/a (Ruderman et al., 2002). Sin embargo, aún no se ha esclarecido el modo en que un dominio incide sobre el otro. En consecuencia, recientemente se han alzado algunas voces (Carlson et al., 2014; Jaga \& Bagraim, 2011) que sostienen que es probable que el impacto del enriquecimiento sea mayor en el rol donde se originaron los recursos que en el rol receptor.

\subsection{Perfiles de CTF y ETF}

La mayoría de las perspectivas tradicionales han visto a la articulación trabajo-familia como a la ausencia de conflicto, o como la frecuencia e intensidad con la que el trabajo interfiere con la familia o la familia con el trabajo (Zhang et al., 2012). A pesar de ello, desde este enfoque la articulación debe ser entendida como las diferentes formas de manifestación cualitativa del CTF y del ETF en la vida de las personas. Las primeras combinaciones estudiadas (Demerouti \& Geurts, 2004) indicaron una tipología de cuatro formas de balance trabajo-familia: a) alto CTF y bajo ETF (interacción negativa, desbalanceado o dañino); b) alto ETF y bajo CTF (interacción positiva, balanceado o beneficioso); c) bajo CTF y bajo ETF (sin interacción, segmentado, pasivo), y d) alto CTF y alto ETF (interacciones positivas y negativas, difuso o activo). Con posterioridad, Mauno, Kinnunen \& Rantanen (2011) identificaron cinco 
perfiles de articulación trabajo-familia: benéfico (alto ETF y EFT; bajo CTF y CFT), pasivo (bajo ETF y EFT; bajo CTF y CFT), activo-1 (alto CTF, ETF y EFT; muy alto CFT), activo-2 (alto CTF, ETF y EFT; moderado CFT) y contradictorio (alto CTF y bajo ETF; bajo CFT y alto EFT)

Sin embargo, investigaciones posteriores (Rantanen, Kinnunen, Mauno, \& Tillemann, 2011; Rantanen et al., 2013) han advertido que estas clasificaciones representan una simplificación de los mecanismos que se ponen en juego en la vida real. En consecuencia, estos autores (Rantanen et al., 2013) han propuesto la existencia de tres tipos de perfiles: benéfico (alto ETF y EFT; bajo CTF y CFT), activo (alto CTF, CFT, ETF y EFT) y contradictorio (alto CTF y bajo ETF; bajo CFT y alto EFT). Los resultados empíricos han demostrado que el tipo de balance benéfico mostró mayor bienestar subjetivo, mientras que el activo alcanzó mayores niveles de vigor en el trabajo, así como más tensiones psicológicas y menor satisfacción con la vida que el tipo contradictorio (Rantanen et al., 2013).

Más recientemente, los resultados obtenidos por Carvalho \& Chambel (2016) han permitido delimitar cuatro perfiles de articulación trabajo-familia: dominación del CTF (alto CTF y bajo ETF), relación moderada (moderado CTF y ETF), moderado ETF (moderado ETF y bajo CTF) y, finalmente, relación completa (alto CTF y ETF). Adicionalmente, estos autores han podido establecer que los diferentes tipos de articulación entre el trabajo y la familia proveen un contexto determinante para la experiencia de bienestar del empleado.

\subsection{Variables antecedentes de la articulación trabajo-familia}

Un área fundamental dentro de las investigaciones sobre la temática ha sido el estudio de sus variables explicativas. Al tratarse de dimensiones ortogonales, con distintos predictores y consecuencias, resulta necesario realizar un desarrollo particular para cada una de ellas (Frone, 2003). En cuanto a las variables antecedentes del CTF, la revisión de la bibliografía consultada ha podido dar cuenta del desarrollo de un gran cuerpo teórico en torno a la cuestión. Con respecto a la vertiente positiva, los hallazgos en torno al ETF todavía son incipientes y no se ha alcanzado un nivel de sistematización similar al del CTF, por lo que se ha sugerido la necesidad de destinar mayores esfuerzos para identificar los mecanismos y variables antecedentes del mismo (Cooklin et al., 2015). En relación al estudio conjunto de ambas, evidencias empíricas sugieren que las estrategias empleadas para la reducción del CTF 
pueden también incrementar el ETF, optimizando la calidad general de la interfase trabajo-familia (Bass \& Grzywacz, 2011).

\subsection{Variables antecedentes del CTF}

Los predictores del CTF pueden ser agrupados en dos grandes categorías generales: a) factores ambientales (laborales y familiares) y b) disposicionales (Frone, 2003). En lo que hace al ambiente, se han identificado a los estresores laborales (conflicto de rol, ambigüedad de rol, sobrecarga de rol, demandas de tiempo), el involucramiento en el rol laboral (involucramiento en el trabajo, interés en el trabajo), el apoyo laboral (apoyo organizacional, del supervisor y de los compañeros de trabajo) y las características del trabajo (variedad de tareas, autonomía) como los principales predictores del CTF. Mientras que los estresores familiares (conflicto de rol, ambigüedad de rol, sobrecarga de rol, demandas de tiempo, demandas parentales, número de hijos o personas a cargo), el apoyo familiar (apoyo marital) y las características de la familia (clima familiar) han sido señalados como antecedentes del CFT (Michel, Kotrba, Mitchelson, Clark, \& Baltes, 2011).

Otros aspectos ambientales recientemente propuestos como predictores del CTF han sido la seguridad laboral y la posibilidad de ocupar una posición de prestigio (Cooklin et al., 2015). Asimismo, la modalidad de contratación ha sido relacionada con los niveles de CTF, en tanto que los trabajadores permanentes exhibieron mayores índices de conflicto (Mauno et al., 2015). Paralelamente, se ha señalado (Burke, Koyuncu, \& Fiksenb, 2013) que las jornadas laborales extensas y la falta de flexibilidad laboral se vinculan con el CTF, a diferencia de políticas de salidas laborales abarcativas (Ruppanner, 2013). Sumado a ello, algunos autores (Allen et al., 2015) han encontrado diferencias trasnacionales en los niveles de CFT. En este sentido, los índices de CFT fueron mayores en culturas colectivistas con una mayor inequidad económica entre los sexos.

Por su parte, las variable disposicionales no han recibido el mismo grado de atención en la bibliografía específica (Frone, 2003). Algunas características de personalidad indicadas como antecedentes del CTF en la bibliografía revisada son el locus de control interno, la afectividad negativa y el neuroticismo (Michel et al., 2011). Asimismo, la extraversión, la autoeficacia (Allen et al. 2015), los valores personales (Chernyak-Hai \& Tziner, 2016) la escrupulosidad y la agradabilidad (Wille, De Fruyt, \& Feys, 2013) han sido relacionados con una mayor tendencia a manejar los problemas y evitar el CTF. 
Adicionalmente, se ha encontrado (Allen et al., 2015) que las dimensiones del CTF (tiempo, tensión y comportamiento) se correlacionan de manera diferente con muchas de estas variables disposicionales.

\subsection{Variables antecedentes del ETF}

A diferencia del CTF, donde las variables predictoras se encuentran claramente definidas en función de las demandas de uno u otro dominio, el ETF es predicho por los recursos que se generan a partir de la participación en dichos espacios (Lingard et al., 2010). Por tanto, se han planteado (Salehi, Rasdi, \& Ahmad, 2015) dos grandes grupos de antecedentes del ETF: a) factores ambientales (laborales y familiares), y b) factores disposicionales.

Dentro de los factores ambientales, se ha reportado que el recibir apoyo por parte de la organización a la que pertenece (Wayne, Casper, Matthews, \& Allen, 2013) o de su supervisor (Baral \& Bhargava, 2010) permite que el empleado desarrolle sentimientos positivos como el ETF. Por otra parte, se ha verificado que la autonomía laboral, los sistemas de apoyo informales (Lo Presti \& Mauno, 2016), el control laboral (Salehi et al., 2015), el sistema de trabajo (Carvalho \& Chambel, 2014) y el compromiso laboral (Hakanen \& Peeters, 2015) también predicen el ETF. En este sentido, se ha indicado (McNall, Nicklin, \& Masuda, 2010; Rastogi et al., 2016) que la flexibilidad laboral, en especial la flexibilidad temporal, es un antecedente importante del ETF. En cuanto al dominio familiar, la predicción del EFT ha sido señalada en función de la cohesión familiar y la satisfacción con las relaciones intra familiares (Carlson et al., 2014). Sumado a ello, se ha propuesto (Cooklin et al., 2015) que el apoyo informal o emocional predice este tipo de enriquecimiento.

En relación a los factores disposicionales, se ha indicado que el enriquecimiento se encuentra influenciado por aspectos tales como la extraversión, la salud (Salehi et al., 2015) y la fortaleza de la propia identidad (Wayne, Randel, \& Stevens, 2006). Sumado a ello, recientemente se ha indicado (McNall, Scott \& Nicklin, 2015) que individuos con una alta afectividad positiva experimentan ETF y EFT, mientras que aquellos con preferencias a la integración y a la permeabilidad (Daniel \& Sonnentag, 2016) tienden a experimentar ETF, pero no EFT. Finalmente, se ha señalado que tanto el vigor (MoazamiGoodarzi, Nurmi, Mauno, \& Rantanen, 2015) como el núcleo de auto-evaluación (McNall et al., 2011) predicen el ETF. 


\subsection{Variables consecuentes de la articulación trabajo-familia}

Los avances vinculados a las variables resultantes de las dimensiones de la interacción trabajo-familia han sido disímiles. Mientras que las consecuencias ligadas al CTF son ampliamente exploradas por los estudiosos de la temática, aquellas relacionadas al ETF no han arribado al mismo nivel de sistematización.

3.10. Variables consecuentes del CTF: Su impacto en el dominio laboral, familiar y la salud del empleado

La prevalencia del CTF y sus consecuencias negativas han sido objeto de preocupación tanto para los investigadores como para los profesionales (Scott et al., 2015). Al respecto, un estudio meta-analítico (Amstad, Meier, Fasel, Elfering, \& Semmer, 2011) ha sugerido que, en general, las investigaciones han reportado más consecuencias del CTF ligadas al dominio original. Por lo tanto, ellas pueden ser clasificadas en: a) relacionadas al dominio laboral o familiar y b) relacionadas con la salud del empleado (Frone, 2003).

Con respecto al primer grupo, se ha indicado (Amstad et al., 2011; Buonocore \& Russo, 2013) que tanto el CTF como el CFT predicen negativamente la satisfacción con la carrera y el compromiso organizacional y, positivamente, el estrés laboral. A pesar de ello, estudios recientes (Chen, Brown, Bowers, \& Chang, 2015; Mauno et al., 2015) han coincidido en señalar que el CTF no se relaciona con los deseos de abandonar la organización, al tiempo que el CFT ha sido relacionado con ausencias injustificadas, tardanzas y un menor rendimiento laboral (Frone, 2003). Por otra parte, se ha señalado (Wang \& Tsai, 2014) que el grado de CFT predice negativamente el rendimiento laboral y, entre sus dimensiones constitutivas, exhibe un mayor impacto sobre los comportamientos de ciudadanía organizacional (Allen et al., 2015). Además, los comportamientos laborales contraproducentes pueden surgir como respuesta emocional negativa ante la percepción de inequidad que los empleados tienen sobre las iniciativas organizacionales para balancear el trabajo y la familia (Beauregard, 2014). En relación al dominio familiar, se ha encontrado que el CTF se vincula negativamente con la satisfacción con la vida (Matthews, Swody, \& Barnes-Farrell, 2012) y con la satisfacción marital (Bagherzadeh et al., 2016; van Steenbergen, Kluwer, \& Karney, 2014) al tiempo que predicen el estrés y el rendimiento familiar (Amstad et al., 2011).

En cuanto a los resultados físicos y psicológicos del CTF, se ha corroborado (Padhi \& Pattnaik, 2013) que los efectos de este tipo de conflicto incluyen 
falta de sueño, hipertensión, depresión y, en algunas ocasiones, abuso de sustancias. Así también, se ha señalado que el incremento en los niveles de CTF contribuye a aumentar las tensiones psicológicas (Ten Brummelhuis \& Bakker, 2012), el cansancio emocional (Leineweber, Baltzer, Hanson \& Westerlund, 2013) y el burnout (Bagherzadeh et al., 2016). En definitiva, el CTF predice negativamente el bienestar (Mauno, Kinnunen, Rantanen, Feldt \& Rantanen, 2012) y la salud del empleado (Leineweber et al., 2013).

3.11. Variables consecuentes del ETF: Su impacto sobre aspectos laborales, no laborales y la salud del empleado

Desde la perspectiva positiva del trabajo-familia, las consecuencias del ETF han sido organizadas en torno a tres categorías (McNall et al., 2010): a) relacionadas con el trabajo y los factores relacionados al lugar de trabajo (satisfacción laboral, compromiso afectivo, intenciones de renuncia, rendimiento laboral); b) no relacionadas con el trabajo, considera el enriquecimiento de la familia y otros factores no laborales, y c) relacionadas con la salud, incluye la salud física y psicológica.

De las tres categorías, los resultados laborales han recibido un mayor grado de atención por parte de los investigadores, probablemente por su valor directo para las organizaciones (Padhi \& Pattnaik, 2013). Entre sus consecuencias, se ha encontrado (Akram, Malik, Nadeem, \& Atta, 2014; Baral \& Bhargava, 2010; Brougham, Haar, \& Roche, 2015; McNall et al., 2015) que el ETF predice un mayor nivel de satisfacción laboral, menores intenciones de renuncia y mayor compromiso organizacional (Timms et al., 2015). Investigaciones recientes han señalado que el ETF tiene un efecto directo sobre la reducción del cansancio laboral (Mauno et al., 2015), el bienestar cultural del lugar de trabajo (Brougham et al., 2015) y la generación de sentimientos positivos hacia el trabajo y la organización (Wayne et al., 2006). Por otra parte, se han encontrado algunos resultados empíricos escasos e inconclusos acerca de la relación entre el ETF y el rendimiento laboral y sus dimensiones (Baral \& Bhargava, 2010; Carlson, Kacmar, Zivnuska, Ferguson, \& Whitten, 2011; Lim, Song, \& Choi, 2012). Esto puede deberse a que, según informan los hallazgos empíricos provenientes de estudios recientes (Ghislieri, Gatti, Molino, \& Cortese, 2017; Odle-Dusseau, Hammer, Crain, \& Bodner, 2016), el vínculo entre ambas se encuentra mediado por el compromiso afectivo.

En contraposición, las consecuencias familiares del ETF han sido ampliamente ignoradas, excepto en el caso de algunos estudios que han evaluado su relación con la satisfacción familiar (Baral \& Bhargava, 2010; Timms et al., 
2015) y marital (van Steenbergen et al., 2014). De esta manera, se ha indicado (Timms et al., 2015) que las experiencias del trabajo que contribuyen a un humor positivo (ETF-afecto) y a un sentido de confianza (ETF-capital) en la vida familiar se asocian a la satisfacción familiar.

En cuanto a los resultados asociados a la salud del empleado, se ha sostenido (Baral \& Bhargava 2010; McNall et al., 2010) que tanto el ETF como el EFT se vinculan positivamente con el bienestar mental y físico del trabajador. De esta manera, el ETF puede generar recursos que resultan esenciales para afrontar los estresores ya sean familiares o laborales (Greenhaus \& Powell, 2006). Además, se ha constatado que ambos tipos de enriquecimiento predicen mayor auto-eficacia (Chan et al., 2016) y niveles de bienestar subjetivo (Padhi \& Pattnaik, 2013). Por lo tanto, promover el ETF de los empleados surge como una intervención prometedora y un enfoque integrado para trabajar cuestiones relacionadas con la salud mental en el lugar de trabajo (Cooklin et al., 2015).

\subsection{Medición de la articulación trabajo-familia}

La evaluación de la articulación trabajo-familia y de sus dimensiones no ha sido un tópico exento de desacuerdos dentro de la bibliografía especializada. En cuanto a la perspectiva negativa, la revisión realizada ha podido dar cuenta de diversos instrumentos que evalúan una o ambas direcciones del CTF y los diferentes tipos de conflicto. Por otra parte, se han relevado escalas para medir las diferentes denominaciones del intercambio positivo entre el trabajo y la familia. $Y$, finalmente, se han encontrado algunos instrumentos destinados a medir ambos aspectos de forma conjunta.

Con respecto al CTF, si bien en su definición se aclara que el funcionamiento familiar puede verse limitado a causa de las responsabilidades laborales o viceversa, las primeras operacionalizaciones del CTF (Adams, King, \& King, 1996; Greenhaus \& Beutell, 1985) no incorporaron la naturaleza bidimensional de la variable (Tement, Korunka, \& Pfifer, 2010). Los ulteriores intentos por superar tales dificultades (Frone, 2003; O'Driscoll, Ilgen, \& Hildreth, 1992) no han seguido un procedimiento riguroso a la hora de desarrollar los instrumentos de medición. Con base en dichos inconvenientes, y a partir de la revisión de estudios previos, Netemeyer, Boles y McMurrian (1996) diseñaron una escala compuesta por 10 ítems (cinco para cada sentido del constructo), valorados en una escala Likert de siete puntos (variando desde $1=$ "fuertemente en desacuerdo" a 7="fuertemente de acuerdo"). Con posterioridad, se encontró (Boyar, Carson, Mosley Jr, Maertz Jr, \& Pearson, 2006) 
que al reducir la cantidad de reactivos del instrumento (cuatro ítems para medir CTF y tres para CFT) se mejoraban sus propiedades psicométricas.

Por su parte, la escala de Carlson et al. (2000) incluyó la perspectiva bidireccional del CTF y, además, tuvo en consideración los diferentes tipos de conflicto. Este instrumento fue realizado a partir de una validación rigurosa en cinco muestras diferentes. Los resultados confirmaron seis dimensiones distintas que incluían las tres formas de CTF (tiempo, tensiones y comportamiento) y sus dos direcciones. La escala en cuestión quedó compuesta por 18 ítems, tres por cada dimensión, valorados en una escala tipo Likert de cinco puntos (variando desde 1="fuertemente en desacuerdo" a $5=$ "fuertemente de acuerdo"). La misma posee una versión abreviada (Matthews, Kath, \& Barnes-Farrell, 2010) y ha sido validada para su uso en otros países (Tement et al., 2010).

En relación a la evaluación de la dimensión positiva de la articulación trabajo-familia, algunas revisiones específicas (Carlson et al., 2006; Greenhaus \& Powell, 2006) han hecho mención a una gran cantidad de instrumentos que carecen de claridad conceptual. En este sentido, se pueden señalar escalas desarrolladas para medir el mejoramiento del rol (Tiedje et al., 1990), el enriquecimiento de recursos (Cohen \& Kirchmeyer, 1995), el derrame positivo (Grzywacz \& Marks, 2000; Kirchmeyer, 1995), y la facilitación trabajo-familia (Grzywacz \& Bass, 2003; Wayne et al., 2006).

Teniendo en cuenta tal proliferación de instrumentos, Greenhaus y Powell (2006) indicaron que sin una clara definición de la vertiente positiva de la articulación trabajo-familia sería muy difícil arribar a un instrumento que abarque el amplio espectro del constructo. Por lo tanto, a partir de la conceptualización del ETF desarrollaron y validaron una escala para evaluarlo. La escala quedó compuesta por 18 ítems que incluían las tres formas de ETF (desarrollo, afecto y capital) y sus dos direcciones. La misma ha sido ampliamente difundida en la bibliografía actual (Akram et al., 2014; Chan et al., 2016; Jijena-Michel \& Jijena-Michel, 2015; Korabik \& Warner, 2013) y cuenta con validaciones en países como Eslovaquia (Tement et al., 2010), Korea (Lim et al., 2012), Portugal (Wayne et al., 2006), Brasil (Gabardo-Martins, Ferreira, \& Valentini, 2016) y Argentina (Omar et al., 2015), entre otros. Sumado a ello, recientemente se ha presentado (Kacmar, Crawford, Carlson, Ferguson, \& Whitten, 2014) una versión reducida del instrumento que contiene sólo tres ítems por cada dirección del enriquecimiento y exhibe propiedades psicométricas similares a la escala completa. 
Finalmente, Geurts et al. (2005) desarrollaron el Cuestionario de Interacción Trabajo-Familia (Survey Work-Home Interaction-Nijmegen, SWING) con una muestra de trabajadores europeos, a partir de la adecuación de un instrumento anterior (Grzywacz \& Marks, 2000). El instrumento original presentaba ciertas limitaciones, como el solapamiento de algunos de los ítems con constructos relacionados como la fatiga o el apoyo familiar. De esta manera, se conformó un cuestionario integrado por 22 ítems valorados en una escala Likert de cuatro puntos (variando desde $0=$ "nunca" a $3=$ "siempre"), que permitía evaluar ambos aspectos del constructo (interacción positiva y negativa) y las dos direcciones posibles de las interacciones (trabajo-familia y familia-trabajo). Dado que se trata de un instrumento teóricamente elaborado y con adecuadas propiedades psicométricas ha sido validado posteriormente en países como Francia (Lourel, Gana \& Wawrzyniak, 2005), España (Moreno Jiménez, Sanz Vergel, Rodríguez Muñoz, \& Geurts, 2009) y Argentina (Gabini, 2017).

\section{DISCUSIONES}

Las alteraciones en los enfoques tradicionales de la organización del trabajo y la familia, desafían a los trabajadores a buscar las posibilidades de balancear sus roles en dos de las esferas más importanes de la vida. En línea con ello, las investigaciones en torno a la temática han exhibido un incremento significativo en las últimas décadas, lo que ha transformado a la articulación trabajo-familia en un objeto de estudio primordial para los investigadores de la psicología organizacional. En este sentido, si bien existe una amplia variedad de miradas en relación al constructo, los estudiosos de la temática (Frone, 2003; Padhi \& Pattnaik, 2013) coinciden en señalar que para capturar la multidimensionalidad del fenómeno se deben tener en cuenta los conceptos de CTF y ETF y la dirección en la que se genera el impacto (trabajo-familia o familia-trabajo). Sin embargo, la revisión de la bibliografía recuperada da cuenta de la persistencia de algunos desacuerdos en relación a: a) el grado de sistematización de las investigaciones sobre cada dimensión, b) la relación entre el CTF y el ETF, (c) la direccionalidad de las consecuencias, y d) su evaluación conjunta.

En cuanto al primer punto, el avance de la literatura específica ha estado ampliamente dominado por la perspectiva del conflicto. A su vez, la inexistencia de un marco teórico integrador para el aspecto positivo del trabajo-familia ha dificultado las investigaciones en el área (Greenhause \& Powell, 2006). Esta situación ha derivado en un avance disímil de los estudios sobre el fenómeno. En este sentido, mientras que las variables antecedentes y conse- 
cuentes ligadas al CTF han sido ampliamente exploradas por los estudiosos de la temática (Scott et al., 2015), aquellas relacionadas al ETF no han arribado al mismo nivel de sistematización. Sin embargo, el análisis de las publicaciones analizadas deja entrever una mayor equidad en cuanto al abordaje de la perspectiva negativa y positiva de la articulación trabajo-familia.

En relación al segundo punto, a pesar que un importante cúmulo de investigaciones ha optado por el abordaje individual de cada una de ellas, la realidad indica que los individuos experimentan simultáneamente CTF y ETF $y$, por lo tanto, no debieran ser estudiados como compartimentos estancos sin interacción. Los resultados obtenidos permiten corroborar un sostenido interés por investigar ambas perspectivas de manera conjunta. Desde este enfoque, la articulación debe ser entendida como las diferentes formas de manifestación cualitativa del CTF y del ETF en la vida de las personas (Padhi \& Pattnaik, 2013). A pesar de ello, ninguna de las combinaciones propuestas hasta la fecha (Demerouti \& Geurts, 2004; Rantanen et al., 2011; Rantanen et al., 2013) ha logrado un consenso dentro la comunidad científica.

En torno a la direccionalidad del CTF se ha demostrado (Carlson et al., 2000) que las consecuencias del CTF pueden darse tanto en el dominio en el que se generan como en el que impactan. Sin embargo, un estudio meta-analítico (Amstad et al., 2011) ha sugerido que, en general, las investigaciones han reportado más consecuencias del CTF ligadas al dominio original. De manera similar, en el caso del ETF, recientemente se ha indicado (Carlson et al., 2014; Jaga \& Bagraim, 2011) que probablemente el impacto del enriquecimiento sea mayor en el rol donde se originaron los recursos que en el rol receptor.

Por último, en lo referente a la evaluación de la articulación trabajo-familia, la revisión bibliográfica ha permitido identificar una serie de instrumentos diseñados para medir las dimensiones del fenómeno de manera individual los que, en muchas ocasiones, no han sido desarrollados a partir de un procedimiento metodológico riguroso (Netemeyer et al., 1996) ni han incorporado la bidireccionalidad de los constructos (Tement et al., 2010). Particularmente, la escala de ETF desarrollada por Greenhaus y Powell (2006) ha sido ampliamente difundida en la bibliografía actual y cuenta con diversas validaciones (Gabardo-Martins et al., 2016; Lim et al., 2012; Omar et al., 2015; Tement et al., 2010; Wayne et al., 2006). En cambio, no se ha podido advertir la misma profusión de instrumentos a la hora de evaluar ambos aspectos del constructo de manera conjunta (interacción positiva y negativa) y sus dos direcciones (trabajo-familia y familia-trabajo), con excepción del cuestionario 
SWING (Geurts et al., 2005) y sus ulteriores validaciones (Gabini, 2017; Lourel et al., 2005; Moreno Jiménez et al., 2009).

Frente a este panorama, futuras investigaciones sobre la temática podrían contemplar, en primer lugar, la necesidad de destinar mayores esfuerzos para identificar y sistematizar las variables antecedentes y consecuentes del ETF (Cooklin et al., 2015). Segundo, avanzar hacia una tipología que incorpore las diversas combinaciones de CTF y ETF ajustada a la realidad y que concite la aceptación de la comunidad científica. Tercero, esclarecer si el dominio receptor de los conflictos o recursos es el mismo dominio en el que se originan y sus implicancias teórico-prácticas. Y, cuarto, incorporar estudios que evalúen CTF y ETF de manera conjunta, ampliar los alcances del modelo para verificar su validez transcultural y validar instrumentos con adecuadas propiedades psicométricas para tales fines.

Por último, la presente revisión presenta algunas limitaciones entre las que se encuentran aquellas derivadas del posible sesgo de selección introducido por los criterios de inclusión definidos, es decir, la inclusión mayoritaria de estudios en inglés y el hecho de acceder exclusivamente a artículos a texto completo de acceso abierto. Sin embargo, y a pesar de tales limitaciones, constituye una puesta al día sobre la problemática de la articulación trabajo-familia.

Financiamiento: El presente trabajo ha sido realizado en el marco de la beca doctoral otorgada al autor por el Consejo Nacional de Investigaciones Científicas y Técnicas (CONICET), y dirigido por la Dra. Alicia Omar (CONICET; Universidad Nacional de Rosario, Argentina).

\section{REFERENCIAS}

Adams, G. A., King, L. A., \& King, D. W. (1996). Relationships of job and family involvement, family social support, and work-family conflict with job and life satisfaction. Journal of Applied Psychology, 81(4), 411-420. http://dx.doi. org/10.1037/0021-9010.81.4.411

Akram, H., Malik, N. I., Nadeem, M., \& Atta, M. (2014). Work-family enrichment as predictors of work outcomes among teachers. Pakistan Journal of Commerce and Social Sciences, 8(3), 733-743. Recovered http://www.jespk.net/publications/205.pdf

Allen, T. D., French, K. A., Dumani, S., \& Shockley, K. M. (2015). Meta-analysis of work-family conflict mean differences: Does national context matter? Journal of Vocational Behavior, 90, 90-100. https://doi.org/10.1016/j.jvb.2015.07.006

Allen, T. D., Johnson, R. C., Kiburz, K. M., \& Shockley, K. M. (2013). Work-family conflict and flexible work arrangements: Deconstructing flexibility. Personnel Psychology, 66(2), 345-376. https://doi.org/10.1111/peps.12012 
Amstad, F. T., Meier, L. L., Fasel, U., Elfering, A., \& Semmer, N. K. (2011). A meta-analysis of work-family conflict and various outcomes with a special emphasis on crossdomain versus matching-domain relations. Journal of Occupational Health Psychology, 16(2), 151-169. https://www.ncbi.nlm.nih.gov/pubmed/21280939

Ashforth, B. E., Kreiner, G. E., \& Fugate, M. (2000). All in a day's work: Boundaries and micro role transitions. Academy of Management Review, 25(3), 472-491. https:// doi.org/10.5465/amr.2000.3363315

Ato, M., López, J. J., \& Benavente, A. (2013). Un sistema de clasificación de los diseños de investigación en psicología. Anales de Psicología, 29(3), 1038-1059. http:// dx.doi.org/10.6018/analesps.29.3.178511

Bagherzadeh, R., Taghizadeh, Z., Mohammadi, E., Kazemnejad, A., Pourreza, A., \& Ebadi, A. (2016). Relationship of work-family conflict with burnout and marital satisfaction: Cross-domain or source attribution relations? Health Promotion Perspectives, 6(1), 31-36. https://www.ncbi.nlm.nih.gov/pubmed/27123434

Bakker, A. B., \& Geurts, S. A. (2004). Toward a dual-process model of work-home interference. Work and occupations, 31(3), 345-366. https://doi. org/10.1177/0730888404266349

Baral, R., \& Bhargava, S. (2010). Work-family enrichment as a mediator between organizationalinterventionsforwork-life balanceandjoboutcomes.JournalofManagerial Psychology, 25(3), 274-300. https://doi.org/10.1108/02683941011023749

Bass, B. L., \& Grzywacz, J. G. (2011). Job adequacy and work-family balance: Looking at jobs as a whole. Journal of Family Issues, 32(3), 317-345. https://doi. org/10.1177/0192513X10384215

Beauregard, T. A. (2014). Fairness perceptions of work-life balance initiatives: Effects on counterproductive work behaviour. British Journal of Management, 25(4), 772-789. https://doi.org/10.1111/1467-8551.12052

Boyar, S. L., Carson, C. M., Mosley Jr, D. C., Maertz Jr, C. P., \& Pearson, A. W. (2006). Assessment of the validity of Netemeyer et al.'s (1996) WFC and FWC scales. International Journal of Conflict Management, 17(1), 34-44. https://doi. org/10.1108/10444060610734163

Brougham, D., Haar, J., \& Roche, M. (2015). Work-family enrichment, collectivism, and workplace cultural outcomes: A study of New Zealand Maori. New Zealand Journal of Employment Relations, 40(1), 19-34. Recovered http://hdl.handle. net/10289/9933

Buonocore, F., \& Russo, M. (2013). Reducing the effects of work-family conflict on job satisfaction: The kind of commitment matters. Human Resource Management Journal, 23(1), 91-108. https://doi.org/10.1111/j.1748-8583.2011.00187.x

Burke, R. J., \& Greenglass, E. (1987). Work and family. In C. L. Cooper \& I. T. Robertson (Eds.). International review of industrial and organizational psychology (273-320). New York: John Wiley \& Sons Inc.

Burke, R. J., Koyuncu, M., \& Fiksenb, L. (2013). Antecedents and consequences of work-family conflict and family-work conflict among frontline employees in Turkish hotels. IUP Journal of Management Research, 12(4), 39-55. Recovered https://ssrn.com/abstract $=2445250$

Carlson, D. S., Hunter, E. M., Ferguson, M., \& Whitten, D. (2014). Work-family enrichment and satisfaction mediating processes and relative impact of originating and receiving domains. Journal of Management, 40(3), 845-865. https:// doi.org/10.1177/0149206311414429 
Carlson, D. S., Kacmar, K. M., \& Williams, L. J. (2000). Construction and initial validation of a multidimensional measure of work-family conflict. Journal of Vocational Behavior, 56(2), 249-276. https://doi.org/10.1006/jvbe.1999.1713

Carlson, D. S., Kacmar, K. M., Wayne, J. H., \& Grzywacz, J. G. (2006). Measuring the positive side of the work-family interface: Development and validation of a work-family enrichment scale. Journal of Vocational Behavior, 68(1), 131-164. https://doi.org/10.1016/j.jvb.2005.02.002

Carlson, D. S., Kacmar, K. M., Zivnuska, S., Ferguson, M., \& Whitten, D. (2011). Work-family enrichment and job performance: A constructive replication of affective events theory. Journal of Occupational Health Psychology, 16(3), 297-312. https://doi.org/10.1037/a0022880

Carvalho, V. S., \& Chambel, M. J. (2014). Work-to-family enrichment and employees' well-being: High performance work system and job characteristics. Social Indicators Research, 119(1), 373-387. https://doi.org/10.1007/s11205-0130475-8

Carvalho, V. S., \& Chambel, M. J. (2016). Work-to-family enrichment and conflict profiles: job characteristics and employees' well-being. The Spanish Journal of Psychology. Advance online publication. https://doi.org/10.1017/sjp.2016.63

Champoux, J. E. (1978). Perceptions of work and nonwork: A reexamination of the compensatory and spillover models. Work and Occupations, 5(4), 402-422. https://doi.org/10.1177/073088847800500402

Chan, X. W., Kalliath, T., Brough, P., Siu, O. L., O’Driscoll, M. P., \& Timms, C. (2016). Work-family enrichment and satisfaction: The mediating role of self-efficacy and work-life balance. The International Journal of Human Resource Management, 27(15), 1-22. https://doi.org/10.1080/09585192.2015.1075574

Chen, I. H., Brown, R., Bowers, B. J., \& Chang, W. Y. (2015). Work-to-family conflict as a mediator of the relationship between job satisfaction and turnover intention. Journal of Advanced Nursing, 71(10), 2350-2363. https://doi.org/10.1111/ jan.12706

Chernyak-Hai, L., \& Tziner, A. (2016). The "I believe" and the "I invest" of work-family balance: The indirect influences of personal values and work engagement via perceived organizational climate and workplace burnout. Revista de Psicología del Trabajo y de las Organizaciones, 32(1), 1-10. https://doi.org/10.1016/j. rpto.2015.11.004

Clark, S. C. (2000). Work/family border theory: A new theory of work/family balance. Human Relations, 53(6), 747-770. https://doi.org/10.1177/0018726700536001

Cohen, A., \& Kirchmeyer, C. (1995). A multidimensional approach to the relation between organizational commitment and nonwork participation. Journal of Vocational Behavior, 46(2), 189-202. https://doi.org/10.1006/jvbe.1995.1012

Cooklin, A. R., Westrupp, E., Strazdins, L., Giallo, R., Martin, A., \& Nicholson, J. M. (2015). Mothers' work-family conflict and enrichment: Associations with parenting quality and couple relationship. Child: Care, Health and Development, 41(2), 266-277. https://doi.org/10.1111/cch.12137

Crain, T. L., \& Hammer, L. B. (2013). Work-family enrichment: A systematic review of antecedents, outcomes, and mechanisms. In Arnold B. Bakker (Ed.). Advances in Positive Organizational Psychology (303-328). Howard House, UK: Emerald Group Publishing Limited.

Daniel, S., \& Sonnentag, S. (2016). Crossing the borders: The relationship between boundary management, work-family enrichment and job satisfaction. The International Journal of Human Resource Management, 27(4), 407-426. https://doi. org/10.1080/09585192.2015.1020826 
Demerouti, E., \& Geurts, S. (2004). Towards a typology of work-home interaction. Community, Work \& Family, 7(3), 285-309. https://doi.org/10.1080/136688004 2000295727

Edwards, J. R., \& Rothbard, N. P. (2000). Mechanisms linking work and family: Clarifying the relationship between work and family constructs. Academy of Management Review, 25(1), 178-199. https://doi.org/10.5465/AMR.2000.2791609

Frone, M. R. (2003). Work-family balance. In J. C. Quick \& L. E. Tetrick (Eds.). Handbook of occupational health psychology (pp. 143-162). Washington, DC: American Psychological Association.

Gabardo-Martins, L. M. D., Ferreira, M. C., \& Valentini, F. (2016). Evidências de validade da Escala de Enriquecimento Trabalho-Família em amostras brasileiras. Revista Psicologia-Teoria e Prática, 18(1), 100-112. http://pepsic.bvsalud.org/ $\mathrm{pdf} / \mathrm{ptp} / \mathrm{v18n} 1 / 08 . \mathrm{pdf}$

Gabini, S. (2017). Interacción trabajo-familia: Adaptación y validación de un instrumento para medirla. Revista da UIIPS, 5(5), 24-32. Recuperado de http://ojs. ipsantarem.pt/index.php/REVUIIPS/article/view/438

Geurts, S. A., Taris, T. W., Kompier, M. A., Dikkers, J. S., Van Hooff, M. L., \& Kinnunen, U. M. (2005). Work-home interaction from a work psychological perspective: Development and validation of a new questionnaire, the SWING. Work \& Stress, 19(4), 319-339. https://doi.org/10.1080/02678370500410208

Ghislieri, C., Gatti, P., Molino, M., \& Cortese, C. G. (2017). Work-family conflict and enrichment in nurses: Between job demands, perceived organisational support and work-family backlash. Journal of Nursing Management, 25(1), 65-75. https:// doi.org/10.1111/jonm.12442

Greenhaus, J. H., \& Beutell, N. J. (1985). Sources of conflict between work and family roles. Academy of Management Review, 10(1), 76-88. https://doi.org/10.5465/ AMR.1985.4277352

Greenhaus, J. H., \& Powell, G. N. (2006). When work and family are allies: A theory of work-family enrichment. Academy of Management Review, 31(1), 72-92. https:// doi.org/10.5465/AMR.2006.19379625

Grzywacz, J. G., \& Bass, B. L. (2003). Work, family, and mental health: Testing different models of work-family fit. Journal of Marriage and Family, 65(1), 248-261. https:// doi.org/10.1111/j.1741-3737.2003.00248.x

Grzywacz, J. G., \& Marks, N. F. (2000). Reconceptualizing the work-family interface: An ecological perspective on the correlates of positive and negative spillover between work and family. Journal of Occupational Health Psychology, 5(1), 111-126. http://www.scielo.org.co/scielo.php?script=sci_nlinks\&ref=000147\&pi$d=S 0121-5469201500010001200024 \&$ Ing=en

Hakanen, J., \& Peeters, M. (2015). How do work engagement, workaholism, and the work-to-family interface affect each other? A 7-year follow-up study. Journal of Occupational and Environmental Medicine, 57(6), 601-609. http://www.scielo.org.co/scielo.php?script=sci_nlinks\&ref=000147\&pi$\mathrm{d}=$ S0121-5469201500010001200024\&Ing=en

Jaga, A., \& Bagraim, J. (2011). The relationship between work-family enrichment and work-family satisfaction outcomes. South African Journal of Psychology, 41(1), 52-62. https://doi.org/10.1177/008124631104100106

Jijena-Michel, R. D., \& Jijena-Michel, C. E. (2015). El rol moderador de la flexibilidad del horario de trabajo en la relación del enriquecimiento trabajo-familia y la satisfacción docente. Horizontes Empresariales, 10(2), 41-56. Recuperado de http:// revistas.ubiobio.cl/index.php/HHEE/article/view/2018 
Kacmar, K. M., Crawford, W. S., Carlson, D. S., Ferguson, M., \& Whitten, D. (2014). A short and valid measure of work-family enrichment. Journal of Occupational Health Psychology, 19(1), 32-45. https://www.ncbi.nlm.nih.gov/pubmed/24447219

Kirchmeyer, C. (1995). Managing the work-nonwork boundary: An assessment of organizational responses. Human Relations, 48(5), 515-536. https://doi. org/10.1177/001872679504800504

Korabik, K., \& Warner, M. (2013). The impact of co-workers on work-to-family enrichment and organizational outcomes. In S. Poelmans, J. Greenhaus, \& M. Las Heras Maestro (Eds.). Expanding the Boundaries of Work-Family Research (pp. 254-276). London: Palgrave Macmillan.

Leineweber, C., Baltzer, M., Hanson, L. L. M., \& Westerlund, H. (2013). Work-family conflict and health in Swedish working women and men: A 2-year prospective analysis. The European Journal of Public Health, 23(4), 710-716. https://doi. org/10.1093/eurpub/cks064

Lim, D. H., Song, J. H., \& Choi, M. (2012). Work-family interface: Effect of enrichment and conflict on job performance of Korean workers. Journal of Management \& Organization, 18(03), 383-397. https://doi.org/10.1017/S1833367200000869

Lingard, H. C., Francis, V., \& Turner, M. (2010). Work-family enrichment in the Australian construction industry: Implications for job design. Construction Management and Economics, 28(5), 467-480. https://doi.org/10.1080/01446190903511268

Lo Presti, A., \& Mauno, S. (2016). Are support and control beneficial stress buffers in the presence of work-family barriers? Findings from Italy. International Journal of Stress Management, 23(1), 44-64. https://www.academia.edu/24723225/A short_valid_predictive_measure_of_work_family_conflict_Item_selection_and_ scale_validation

Lourel, M., Gana, K., \& Wawrzyniak, S. (2005). Home-work interaction: A French adaptation and validation of "Survey Work-Home Interaction-Nijmegen"(SWING). Psychologie du Travail et des Organisations, 11(4), 227-239. http://dx.doi. org/10.1016/j.pto.2005.10.003

Matthews, R. A., Kath, L. M., \& Barnes-Farrell, J. L. (2010). A short, valid, predictive measure of work-family conflict: Item selection and scale validation. Journal of Occupational Health Psychology, 15(1), 75-90. https://doi.org/10.1037/a0017443

Matthews, R. A., Swody, C. A., \& Barnes-Farrell, J. L. (2012). Work hours and work-family conflict: The double-edged sword of involvement in work and family. Stress and Health, 28(3), 234-247. https://doi.org/10.1002/smi.1431/full

Mauno, S., De Cuyper, N., Kinnunen, U., Ruokolainen, M., Rantanen, J., \& Mäkikangas, A. (2015). The prospective effects of work-family conflict and enrichment on job exhaustion and turnover intentions: Comparing long-term temporary vs. permanent workers across three waves. Work \& Stress, 29(1), 75-94. https://doi. org/10.1080/02678373.2014.1003997

Mauno, S., Kinnunen, U., \& Rantanen, M. (2011). Work-family conflict and enrichment and perceived health: Does type of family matter? Family Science, 2(1), 1-12. https://doi.org/10.1080/19424620.2011.585908

Mauno, S., Kinnunen, U., Rantanen, J., Feldt, T., \& Rantanen, M. (2012). Relationships of work-family coping strategies with work-family conflict and enrichment: The roles of gender and parenting status. Family Science, 3(2), 109-125. https://doi. org/10.1080/19424620.2012.707618

McNall, L. A., Masuda, A. D., Shanock, L. R., \& Nicklin, J. M. (2011). Interaction of core self-evaluations and perceived organizational support on work-to-family enrichment. The Journal of Psychology, 145(2), 133-149. https://doi.org/10.1080 /00223980.2010.542506 
McNall, L. A., Nicklin, J. M., \& Masuda, A. D. (2010). A meta-analytic review of the consequences associated with work-family enrichment. Journal of Business and Psychology, 25(3), 381-396. https://doi.org/10.1007/s10869-009-9141-1

McNall, L. A., Scott, L. D., \& Nicklin, J. M. (2015). Do positive affectivity and boundary preferences matter for work-family enrichment? A study of human service workers. Journal of Occupational Health Psychology, 20(1), 93-104. https://doi. org/10.1037/a0038165

Michel, J. S., \& Clark, M. A. (2009). Has it been affect all along? A test of work-tofamily and family-to-work models of conflict, enrichment, and satisfaction. Personality and Individual Differences, 47, 163-168. http://dx.doi.org/10.1016/j. paid.2009.02.015

Michel, J. S., Kotrba, L. M., Mitchelson, J. K., Clark, M. A., \& Baltes, B. B. (2011). Antecedents of work-family conflict: A meta-analytic review. Journal of Organizational Behavior, 32(5), 689-725. https://doi.org/10.1002/job.695/full

Moazami-Goodarzi, A., Nurmi, J. E., Mauno, S., \& Rantanen, J. (2015). Cross-lagged relations between work-family enrichment, vigor at work, and core self-evaluations: A three-wave study. Journal of Business and Psychology, 30(3), 473-482. https://doi.org/10.1007/s10869-014-9376-3

Moreno Jiménez, B., Sanz Vergel, A. I., Rodríguez Muñoz, A., \& Geurts, S. (2009). Propiedades psicométricas de la versión española del Cuestionario de Interacción Trabajo-Familia (SWING). Psicothema, 21(2), 331-337. Recuperado de https:// ueaeprints.uea.ac.uk/id/eprint/49328

Morf, M. (1989). The work/life dichotomy. Westport, CT: Quorum.

Netemeyer, R. G., Boles, J. S., \& McMurrian, R. (1996). Development and validation of work-family conflict and family-work conflict scales. Journal of Applied Psychology, 81(4), 400-410. http://dx.doi.org/10.1037/0021-9010.81.4.400

Nicklin, J. M., \& McNall, L. A. (2013). Work-family enrichment, support, and satisfaction: A test of mediation. European Journal of Work and Organizational Psychology, 22(1), 67-77. https://doi.org/10.1080/1359432X.2011.616652

Odle-Dusseau, H. N., Hammer, L. B., Crain, T. L., \& Bodner, T. E. (2016). The influence of family-supportive supervisor training on employee job performance and attitudes: An organizational work-family intervention. Journal of Occupational Health Psychology, 21(3), 296-308. https://doi.org/10.1037/a0039961

O'Driscoll, M. P., llgen, D. R., \& Hildreth, K. (1992). Time devoted to job and off-job activities, interrole conflict, and affective experiences. Journal of Applied Psychology, 77(3), 272-279. http://dx.doi.org/10.1037/1076-8998.7.2.99

Omar, A., Urteaga, F., \& Salessi, S. (2015). Propiedades psicométricas de la Escala de Enriquecimiento Trabajo-Familia para la población argentina. Revista de Psicología, 24(2), 1-18. https://doi.org/10.5354/0719-0581.2015.37689

Padhi, M., \& Pattnaik, S. (2013). A typology of work family research: Perspectives from literature. Vilakshan: The XIMB Journal of Management, 10(2), 83-98. Recovered http://hib.xub.edu.in/Hibiscus/Pub/faccvDet.php?client=xu\&facid=XUF2072

Perestelo-Pérez, L. (2013). Standards on how to develop and report systematic reviews in Psychology and Health. International Journal of Clinical and Health Psychology, 13(1), 49-57. https://doi.org/10.1016/S1697-2600(13)70007-3

Piotrkowski, C. S. (1979). Work and the family system. USA: Free Press; Collier Macmillan.

Powell, G. N., \& Greenhaus, J. H. (2010). Sex, gender, and decisions at the family $\rightarrow$ work interface. Journal of Management, 36(4), 1011-1039. https://doi. org/10.1177/0149206309350774 
Rantanen, J., Kinnunen, U., Mauno, S., \& Tement, S. (2013). Patterns of conflict and enrichment in work-family balance: A three-dimensional typology. Work \& Stress, 27(2), 141-163. https://doi.org/10.1080/02678373.2013.791074

Rantanen, J., Kinnunen, U., Mauno, S., \& Tillemann, K. (2011). Introducing theoretical approaches to work-life balance and testing a new typology among professionals. In S. Kaiser, M. J. Ringlstetter, D. R. Eikhof, \& M. P. Cunha. Creating Balance? (27-46). Berlin: Springer.

Rastogi, M., Rangnekar, S., \& Rastogi, R. (2016). Flexibility as a predictor of work-family enrichment. Global Journal of Flexible Systems Management, 17(1), 5-14. https:// www.researchgate.net/publication/270330275_Benefits_of_Multiple_Roles_ for_Managerial_Women

Romeo, M., Berger, R., Yepes-Baldó, M., \& Ramos, B. (2014). Adaptación y validación de la versión española de la "Survey Work-Home Interaction-NijmeGen" (SWING) en países hispanohablantes. Anales de Psicología, 30(1), 287-293. https://doi. org/10.6018/analesps.30.1.148291

Ruderman, M. N., Ohlott, P. J., Panzer, K., \& King, S. N. (2002). Benefits of multiple roles for managerial women. Academy of Management Journal, 45(2), 369-386. https://www.researchgate.net/publication/270330275_Benefits_of_Multiple_ Roles_for_Managerial_Women

Ruppanner, L. (2013). Conflict between work and family: An investigation of four policy measures. Social Indicators Research, 110(1), 327-347. https://doi. org/10.1007/s11205-011-9933-3

Salehi, P., Rasdi, R. M., \& Ahmad, A. (2015). Personal and environmental predictors of academics' work-to-family enrichment at research universities. The Asia-Pacific Education Researcher, 24(2), 379-388. https://doi.org/10.1007/s40299-0140190-5

Scott, K. L., Ingram, A., Zagenczyk, T. J., \& Shoss, M. K. (2015). Work-family conflict and social undermining behaviour: An examination of PO fit and gender differences. Journal of Occupational and Organizational Psychology, 88(1), 203-218. https://doi.org/10.1111/joop.12091

Staines, G. L. (1980). Spillover versus compensation: A review of the literature on the relationship between work and nonwork. Human Relations, 33(2), 111-129. https://doi.org/10.1177/001872678003300203

Tement, S., Korunka, C., \& Pfifer, A. (2010). Toward the assessment of the work-family interface: Validation of the Slovenian versions of work-family conflict and work-family enrichment scales. Horizons of Psychology, 19, 53-74. Recovered http://psiholoska-obzorja.si/arhiv_clanki/2010_3/tement_et_al.pdf

Ten Brummelhuis, L. L., \& Bakker, A. B. (2012). A resource perspective on the work-home interface: The work-home resources model. American Psychologist, 67(7), 545-556. https://www.ncbi.nlm.nih.gov/pubmed/22506688

Tiedje, L. B., Wortman, C. B., Downey, G., Emmons, C., Biernat, M., \& Lang, R. (1990). Women with multiple roles: Role-compatibility perceptions, satisfaction, and mental health. Journal of Marriage and the Family, 52, 63-72. Recovered https:// www.jstor.org/stable/352838

Timms, C., Brough, P., O’Driscoll, M., Kalliath, T., Siu, O. L., Sit, C., \& Lo, D. (2015). Positive pathways to engaging workers: work-family enrichment as a predictor of work engagement. Asia Pacific Journal of Human Resources, 53(4), 490-510. https://doi.org/10.1111/1744-7941.12066/full

Van Steenbergen, E. F., Kluwer, E. S., \& Karney, B. R. (2014). Work-family enrichment, work-family conflict, and marital satisfaction: A dyadic analysis. Journal of Occupational Health Psychology, 19(2), 182-194. https://doi.org/10.1037/a0036011 
Voydanoff, P. (2004). Implications of work and community demands and resources for work-to-family conflict and facilitation. Journal of Occupational Health Psychology, 9(4), 275. http://dx.doi.org/10.1037/1076-8998.9.4.275

Wang, M. L., \& Tsai, L. J. (2014). Work-family conflict and job performance in nurses: the moderating effects of social support. Journal of Nursing Research, 22(3), 200-207. https://www.ncbi.nlm.nih.gov/pubmed/24730427

Wayne, J. H., Casper, W. J., Matthews, R. A., \& Allen, T. D. (2013). Family-supportive organization perceptions and organizational commitment: The mediating role of work-family conflict and enrichment and partner attitudes. Journal of Applied Psychology, 98(4), 606-622. https://www.ncbi.nlm.nih.gov/pubmed/23565896

Wayne, J. H., Randel, A. E., \& Stevens, J. (2006). The role of identity and work-family support in work-family enrichment and its work-related consequences. Journal of Vocational Behavior, 69, 445-461 http://dx.doi.org/10.1016/j.jvb.2006.07.002

Wille, B., De Fruyt, F., \& Feys, M. (2013). Big five traits and intrinsic success in the new career era: A 15-Year longitudinal study on employability and work-family conflict. Applied Psychology, 62(1), 124-156. https://doi.org/10.1111/j.14640597.2012.00516.x

Zhang, H., Yip, P. S., Chi, P., Chan, K., Cheung, Y. T., \& Zhang, X. (2012). Factor structure and psychometric properties of the Work-Family Balance Scale in an urban Chinese sample. Social Indicators Research, 105(3), 409-418. https://doi. org/10.1007/s11205-010-9776-3

\section{(cc) BY}

